\title{
MigRACIONES Y PERCEPCIÓN DE SEGURIDAD ALIMENTARIA: EL CASO DE LA CULTURA ALIMENTARIA DE LAS MUJERES MATSIGENKA de la Comunidad Nativa Nuevo Mundo (Megantoni, Cusco)
}

\author{
Ruth Sebastián Leonidas \\ Universidad Nacional Mayor de San Marcos \\ musa_mylove@hotmail.com \\ (1) https://orcid.org/0000-0002-1844-6575
}

\section{Resumen}

Esta investigación busca comprender los procesos migratorios ocurridos en los últimos veinte años y explorar la percepción de seguridad alimentaria para explicar su influencia en la cultura alimentaria de las mujeres matsigenka de la comunidad nativa Nuevo Mundo (Megantoni, Cusco). De esta manera, uno de los objetivos específicos de esta investigación es describir las historias migratorias de las familias matsigenka para poder explicar su influencia en los cambios de sus hábitos y dietas alimenticios, así como explorar y conocer la influencia de la percepción de seguridad alimentaria en la práctica en los hábitos alimentarios de las mujeres de la comunidad, para finalmente explicar la construcción de la identidad y las relaciones de género a partir de la alimentación en la comunidad.

Palabras claves: Matsigenka; Procesos migratorios; Seguridad alimentaria; Relaciones de género 


\section{Summary}

This research proposal seeks to understand the migratory processes that have occurred in the last twenty years and to explore the perception of food security to explain its influence on the dietary culture of matsigenka women of the Nuevo Mundo native community (Megantoni, Cusco). One of the specific objectives of this research project is to describe the migratory history of matsigenka families in order to explain its influence on changes in their habits and content of consumption as well as exploring and understanding the influence of the perception of food security on the habits of women in the community in order to explain the construction of identity and gender relations related to food in the community.

Keywords: Matsigenka; Migratory processes; Food security; Gender relations

\section{El problema de investigación}

Este es un proyecto de investigación para optar el título de antropóloga en la Universidad Nacional Mayor de San Marcos. Las comunidades nativas del distrito de Megantoni (Cusco) están atravesando cambios en su organización social comunal, relaciones sociales de género, identidad, imagen del cuerpo y la división sexual del trabajo acaecidos dentro de un proceso de modernización y mercado que ha conectado la localidad con la sociedad mayor, la peruana. Es en ese sentido que estudiar la alimentación de las poblaciones del distrito es importante ya que devela los cambios que están ocurriendo a nivel macro en las instituciones sociales como en las micro (marcos simbólicos de significados) relacionados a la comida y los alimentos. Respecto a la comunidad matsigenka de Nuevo Mundo, siendo una de las comunidades del distrito que está atravesando esos cambios, es pertinente abordar la alimentación en el sentido de que se puede comprender los procesos migratorios ocurridos en los últimos veinte años en el distrito y al mismo tiempo permite explorar la percepción de seguridad alimentaria para explicar su influencia en la cultura alimentaria de las mujeres matsigenka de la comunidad nativa Nuevo Mundo (Megantoni, Cusco). Entonces, dentro de un contexto de modernización se cuestiona las reconfiguraciones y cambios en los hábitos alimenticios en relación a lo que se entiende, entre las mujeres matsigenka, la noción de seguridad alimentaria. Es decir, cómo se manifiesta esta seguridad alimentaria en la práctica de los hábitos alimenticios en la comunidad de estudio. Relacionarlo pues con ese proceso múltiple y fragmentado en donde las mujeres matsigenka construyen, producen y reproducen sus identidades colectivas 
e individuales, siempre dinámicas, en base a los imaginarios sociales y culturales. Entonces hay un mestizaje culinario en base a los platos y comidas del día de las familias matsigenka, donde los alimentos de la chacra como la yuca y las diferentes variedades de pescado (sábalo, paiche, doncella, carachama, etc.) se han complementado con los productos procesados e industrializados como el aceite, el azúcar, el arroz, los huevos, y estos han formado platos combinados.

\section{Justificación}

La investigación busca comprender los procesos migratorios ocurridos en los últimos veinte años, y explorar la percepción de seguridad alimentaria para explicar su influencia en la cultura alimentaria de las mujeres matsigenka de la comunidad nativa Nuevo Mundo (Megantoni, Cusco). Esto permitirá comprender los marcos interpretativos y referencias de la alimentación puestas en práctica en la cotidianidad de las mujeres de la comunidad dentro de los procesos de modernización y mercado que atraviesa la zona.

Es necesario señalar mi papel como investigadora que me hace al mismo tiempo parte de la comunidad de estudio por mis vínculos parentales ya que evidencia mi postura personal frente a la problemática en torno a las migraciones y la percepción de seguridad alimentaria en relación a la cultura alimentaria practicada actualmente en la comunidad. Además, considero pertinente comprender los actuales problemas alimentarios desde una mirada etnográfica tomando los elementos emic y étic como interdependientes entre sí. En lo que sigue se menciona las preguntas y objetivos que serán desarrollados en la presente investigación previa al trabajo de campo.

\section{Pregunta general}

- ¿Cómo los procesos de migración ocurridos en los últimos veinte años y la percepción de seguridad alimentaria influyen en la cultura alimentaria de las mujeres matsigenka de la comunidad nativa Nuevo Mundo (Megantoni, Cusco)?

\section{Preguntas específicas}

- ¿De qué manera las historias migratorias de las familias matsigenka influyen en los cambios de sus hábitos y dietas alimenticias?

- ¿De qué manera la percepción de seguridad alimentaria influye en la práctica en los hábitos alimentarios de las mujeres de la comunidad? 
- ¿Cómo se construyen la identidad y las relaciones de género a partir de la alimentación en la comunidad?

\section{Objetivo general}

- Comprender los procesos migratorios ocurridos en los últimos veinte años y explorar la percepción de seguridad alimentaria para explicar su influencia en la cultura alimentaria de las mujeres matsigenka de la comunidad nativa Nuevo Mundo (Megantoni, Cusco).

\section{Objetivos específicos}

- Describir las historias migratorias de las familias matsigenka para explicar su influencia en los cambios de sus hábitos y dietas alimenticias.

- Explorar y conocer la influencia de la percepción de seguridad alimentaria en la práctica en los hábitos alimentarios de las mujeres de la comunidad.

- Explicar la construcción de la identidad y las relaciones de género a partir de la alimentación en la comunidad.

\section{Estado de la cuestión y marco teórico}

\section{Estado de la cuestión}

La revisión de literatura del proyecto de investigación previa al trabajo de campo ha identificado seis enfoques teóricos. El primer enfoque sobre la alimentación como indicador de identidad refiere que el acto de alimentarse es un fenómeno transversal y biopsicosocial que abarca un sistema de creencias y representaciones simbólicas complejas de la alimentación que va desde la vida cotidiana hasta atravesar diversos ámbitos sociales de la vida humana (Contreras y Gracia 2005; Viola 2008; Cánepa, Hernández, Biffi y Zuleta 2011). En ese sentido, abordar la alimentación partiendo desde los indicadores gustativos de un grupo específico nos puede indicar la particularidad a una identidad culinaria aunado a una delimitación territorial (Contreras y Gracia 2005; Bertran 2010). Es decir, cuando consumo aquello me diferencio de los demás y adopto una identidad hacia un grupo social específico asentado dentro de un territorio (Contreras y Gracia 2005). Y así se construyen esas identidades a través de estas prácticas alimentarias generadas en cada sector social, me identifico por 
qué acepto comer socialmente ciertos alimentos de mi grupo social específico (Entrena 2008). El segundo enfoque teórico es la chacra como sistema agroforestal tradicional, donde la chacra es el hogar de diversos policultivos de varias frutales, constituyendo un sistema de producción agroforestal tradicional y diverso (Cavalcante y G., Lemos, Da Costa, Da Cunha y Barbosa, 2012; Vargas, Prado, Nicolalde, Casanoves, Virginio y Viera 2018). Entonces en muchos estudios amazónicos se afirma que la chacra es el sistema agroforestal más antiguo que las poblaciones indígenas de la Amazonía han sabido aprovechar de su medio para cultivar diversos policultivos de frutales y otros actuales monocultivos de cacao, arroz, algodón, etc., para su consumo propio y/o para la venta (Vargas et al.2018). Estos estudios tienen en cuenta que la sedimentación anual condiciona los sistemas agrícolas, en las varzeas y barreales porque a veces hace perder el cultivo de manera repentina por los repiquetes (julio, agosto y octubre); es decir, las variaciones como lo llaman condicionan la agrobiodiversidad en playas y orillas de ríos (Ríos y Camacho 2016).

El tercer enfoque teórico que se encontró en la revisión de literatura son las políticas públicas y la seguridad alimentaria, donde se argumenta que se debe tener en cuenta las racionalidades simbólicas y conocimientos locales a la hora de implementar políticas públicas en materia de alimentación para las poblaciones que las necesita (Segura 2013; Lee, Paredes, Rengifo, Ambikapathi, Peñataro, Kosek y Caulfield 2014; Oseguera 2004; Morales y Paipilla 2020). Es decir, cuando se hable de seguridad alimentaria en los proyectos de desarrollo impuestas en una comunidad local es imperativo hacerlo dentro de las realidades locales y conocimientos étnicos de la misma población para solucionar sus problemas alimentarios (Morales y Paipilla 2020). Y un cuarto enfoque relacionado a las mujeres como gestoras del cambio, donde se sustenta que somos las mujeres las receptoras de manera generacional de los conocimientos culinarios a través de nuestras abuelas y madres y conforme al contexto en el que estamos viviendo, vamos adecuando los conocimientos alimentarios de acuerdo a las necesidades de la familia en el día a día (Balderrama, Guzmán, Romero y Galván 2018; Troncoso, Muñoz, Sotomayor, Amaya, Alarcos y Amaya 2019). Tanto autores como García (2010) y Abanto (2012), citados por Balderrama et al. (2018) afirman pues que la mujer tiene a su cargo no solo el cuidado de sus hijos (como dedicarse a los quehaceres del hogar) sino también la encargada de proveer y distribuir los alimentos en la familia; obviamente sin olvidarnos del papel que juegan los esposos en este proceso alimentario. 
El enfoque sobre los cambios en los hábitos alimenticios, donde estos se producen por las consecuencias de diferentes intervenciones en las fases del sistema alimentario que pasa por la obtención y producción hasta llegar a la fase del consumo, donde participan diversos actores e instituciones, configurando los patrones para la conformación de hábitos de alimentación de las personas (Castillo y Ayala 2013; Davidson 2018). Es justamente el ingreso del mercado en las comunidades amazónicas y dentro de un contexto globalizador donde se gestan estos cambios en la misma dinámica interna de cada comunidad afectada (Oseguera y Esparza 2009; Davidson 2018). Como último enfoque se encontró a las alternativas alimentarias frente a los procesos globalizadores, donde los autores refieren que ante procesos modernizadores, neoliberales en la alimentación, han surgido nuevas alternativas que luchan y ofrecen alternativas sostenibles de la misma en conjunción con la naturaleza (Oyola y Mosqueira 2013, Segura 2013, Centejas 2014).

\section{Marco teórico-conceptual}

La definición que se manejaba en los 50 y 60 de seguridad alimentaria estaba relacionado con el aprovisionamiento de los alimentos; es decir, entiendo acá el almacenamiento de alimentos por su escasez (Contreras 2005). Conforme entramos al siglo XXI, el significado de la seguridad alimentaria ha ido cambiando, ya no se relacionaba más con el aprovisionamiento de alimentos sino con el consumo de alimentos libre de riesgos presentes en la producción alimentaria industrializada. En otras palabras, se empezaron a tomar precauciones contra los alimentos que se consumían para minimizar los posibles peligros asociados a los alimentos como la intoxicación y la contaminación (Contreras 2005).

Los sistemas de representación simbólica de los alimentos está en una lenta evolución de los sistemas de producción-distribución de los alimentos en el que participan la producción, procesamiento, almacenamiento, transporte, distribución, comercialización y consumo de esos alimentos (Contreras 2005). Esto está relacionado con los gustos/preferencias y rechazos de ciertos alimentos que obedecen a los marcos normativos simbólicos estructurales que obligan e imponen a los comensales los significados de los alimentos que consumen (Conteras 2005). Desde la antropología de la alimentación, el sabor no solo es eso, sino que está compuesto por una compleja red de texturas, colores, olores, temperaturas en relación al alimento y las memorias/sentimientos que estos evocan (Mintz 2003). Tres cosas me quedaron claras aquí: lo primero tiene que ver con que la percepción de los riesgos alimentarios debe ser explicada en los marcos 
culturales caracterizados por los valores sociales y morales determinados por las concepciones del cuerpo y de la imagen corporal y una visión de la enfermedad y la salud. Entonces estamos hablando de la reticencia a asumir riesgos innecesarios si no se perciben ventajas al consumir dicho producto alimentario sospechoso generándose así una sustitución por otro objeto alimentario (Contreras 2005). De ahí que hay dos elementos claves en la capacidad o intención de asumir riesgos innecesarios: la evitabilidad y la imposibilidad de sustitución porque tengamos en cuenta que hay tres diferentes tipos de riesgos: los accidentales y evitables pero ineludibles en la práctica y los provocados (estos últimos por prácticas descuidadas e ineficaces o fraudulentas) (Contreras 2005).

\section{Diseño metodológico}

Esta investigación usó el método etnográfico para recoger información sobre los procesos migratorios ocurridos en los últimos veinte años y explorar la percepción de seguridad alimentaria para explicar su influencia en la cultura alimentaria de las mujeres matsigenka de la comunidad nativa Nuevo Mundo (Megantoni, Cusco). Para ello, las técnicas de investigación usadas en este trabajo fueron la libreta de campo, la observación participante, las conversaciones, las entrevistas abiertas e historia de vida.

\section{Recojo de información}

El trabajo de campo se realizó el 2020 y las técnicas de investigación fueron la libreta de campo, que sirvió de guía para anotar las vivencias etnográficas con las mujeres que fueron protagonistas del presente proyecto. La observación participante fue de utilidad para registrar y contrastar la conducta alimentaria de las entrevistadas en relación con las prácticas culinarias y alimentarias de las mismas. En relación a las conversaciones, serán tomadas en cuenta para profundizar en temáticas en la vida cotidiana que las entrevistas abiertas no podrán abordarse dependiendo de la disposición del tiempo de las mujeres entrevistadas. Respecto a las entrevistas abiertas, se usaron para recoger información sobre temáticas puntuales tales como la historia migratoria de las familias matsigenkas, la noción/percepción de la seguridad alimentaria, la imagen corporal, los hábitos alimenticios, las prácticas culinarias y alimentarias enmarcados en las actividades cotidianas de la casa, chacra y fuera de ellos en la comunidad. Finalmente, la historia de vida fue utilizada para organizar en grupos etarios la percepción de la seguridad alimentaria y hábitos alimenticios entre las mujeres entrevistadas, 
contrastando conductas alimentarias según ámbitos generacionales. Además, como contraparte de la mirada de mujeres, también en menor medida se recogieron opiniones de los hombres de la comunidad.

\section{Análisis de datos}

La codificación y sistematización de los materiales de campo se realizará de la siguiente manera: la información recogida en el trabajo de campo previsto para el año 2020 será codificada preliminarmente siguiendo a tres autores. Primero transcribiendo los materiales, armando los libros de códigos respectivos, así como un libro de código general y un análisis preliminar de dichos códigos (Fernández 2006). Este ejercicio de codificación preliminar durante el trabajo de campo es parte de la primera etapa en el análisis de datos cualitativos que ayudará a detectar temáticas nuevas que irán surgiendo a través de la transcripción y de la lectura en profundidad de los materiales (Gonzales y Arana 2010). Y de esta manera me ayudará a identificar los patrones que emergerán en la codificación de datos (Bernard 1995). Para esto usaré un cuadro donde separaré en dos secciones las afirmaciones, actividades y conductas observadas que podrán ser voluntarias en cualquiera de las situaciones donde se aplicarán las técnicas de investigación que serán usadas para este proyecto. Entonces como temáticas generales que se plasmarán en las guías de las técnicas de investigación giran en torno a la historia migratorias de las entrevistadas, la noción/percepción de la seguridad alimentaria, la conducta alimentaria, los hábitos alimentarios, la imagen corporal y las prácticas culinarias y alimentarias realizadas en las actividades cotidianas de las mujeres enmarcadas dentro de un contexto de globalización y mercado en la que atraviesa la comunidad de estudio.

\section{Estrategia operativa}

\section{Lugar del trabajo}

El área de estudio del presente proyecto de investigación es la comunidad nativa de Nuevo Mundo. Dicha comunidad está ubicada en la margen derecho del río Urubamba en el distrito de Megantoni de la provincia de La Convención en el departamento cusqueño. La comunidad se creó el 15 de setiembre de 1970. Las actividades productivas se despliegan desde los trabajos municipales, las proveedurías de las obras municipales hasta las actividades de subsistencia de caza, pesca y recolección que se practican en menor medida en la actualidad. 
Según la base de datos de pueblos originarios del Ministerio de Cultura, en total hay 11 comunidades nativas y dos asentamientos rurales en el distrito de Megantoni. Estas comunidades nativas tienen tres organizaciones indígenas y están distribuidas de la siguiente manera: Central de Comunidades Nativas Matsiguengas (CECONAMA) tiene en su ala a seis comunidades nativas tales como Segakiato, Camisea, Puerto Huallana, Nuevo Mundo, Ticumpinia y Nueva Vida; el Consejo Machiguenga del Río Urubamba (COMARU) a tres de ellas, Kirigueti, Shivankoreni y Cashiriari y, FECONAYY, a dos, Miaría y Sensa. Y los asentamientos rurales son Shintorini y Túpac Amaru.

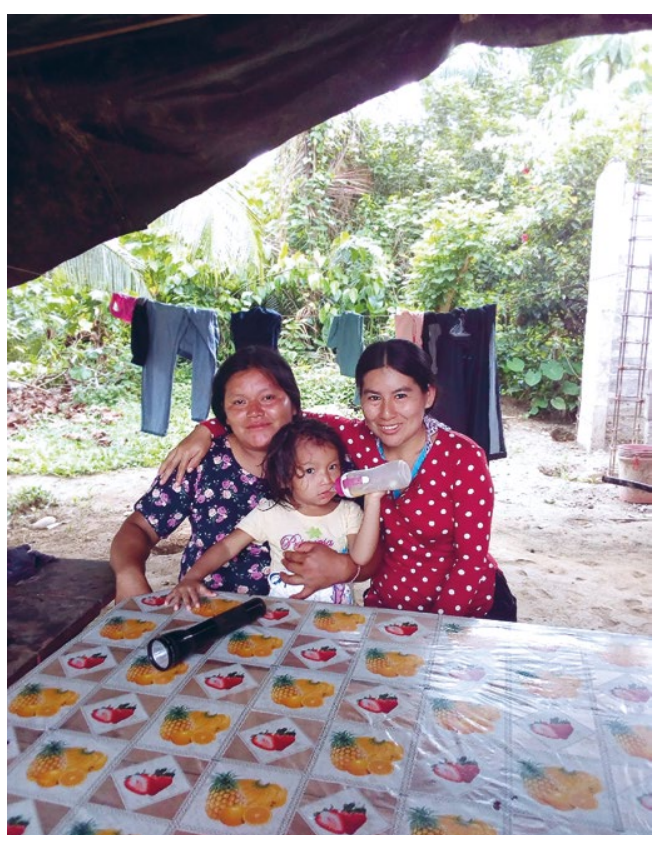

Ruth Sebastián con una informante. 
Ruth Sebastián con sus primas en la chacra.

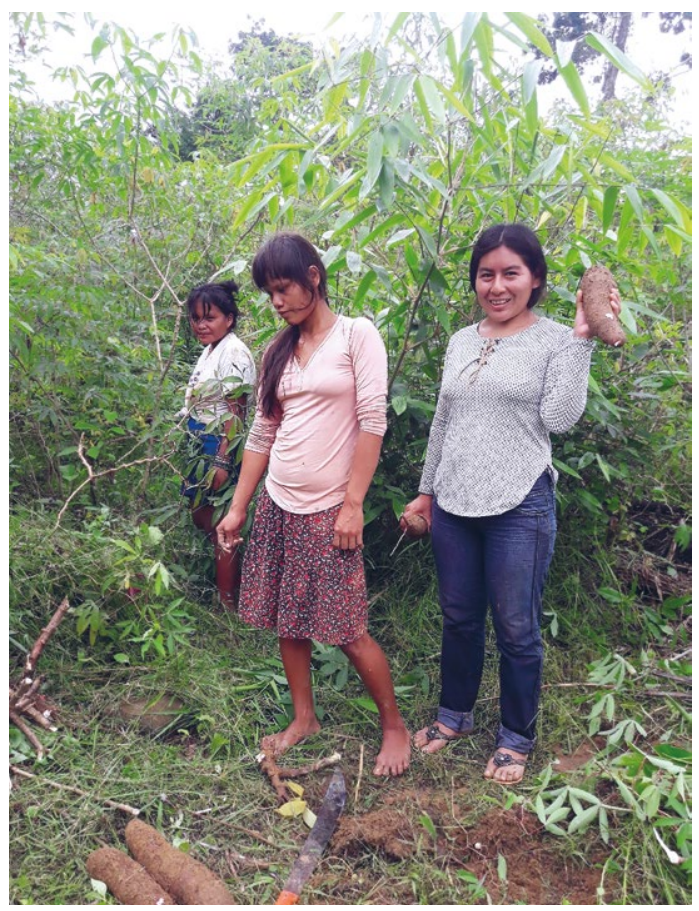




\section{Referencias bibliográficas}

Balderrama Diaz, Raquel; Guzman Saldaña, Rebeca; Romero Palencia, Angélica y Galván, Marcos. "Prácticas alimentarias de mujeres indígenas en función del estado nutricio y sexo de sus hijos/as". En: Revista Mexicana de Trastornos Alimentarios 9(2) (2018): 183-195.

Bernard, Russell H. Métodos de investigación en Antropología. Abordajes cualitativos y cuantitativos. 2da ed. Altamira Press, 1995, 260-293.

Bertran Vila, Miriam. Acercamiento antropológico de la alimentación y salud en México. México: Universidad Autónoma Metropolitana, 2010, 387-410.

Cánepa Coch, Gisela; Hernández Macedo, Miguel; Biffi Isla, Valeria y Zuleta Garcia, Mario. Cocina e identidad: la culinaria peruana como patrimonio cultural inmaterial. 1ra ed. Lima: Ministerio de Cultura, 2011.

Cartay, Rafael. Los regímenes alimentarios de las comunidades indígenas amazónicas y sus impactos medioambientales. Ecuador: Universidad Técnica de Manabí (UTM), 2018.

Castillo Girón, Víctor Manuel y Ayala Ramírez, Suhey. "Hábitos alimentarios y abasto de alimentos en América, Jalisco, México”. En: Espacio Abierto 21(3). Venezuela: Universidad del Zulia, 2012, 452-479.

Cavalcante Martins, Mariana; G. L. F. Veras, Joelna Eline; Lemos Uchoa, Lanaiana; Da Costa Pinheiro, Patricia Neyva; Da Cunha Vieira, Neiva Francenely y Barbosa Ximenes, Lorena. "Food safety and the use of regional food: the validation of a serial álbum”. En: Rev. Esc. Enferm. USP, 46(6) (2012): 1355-1362.

Centejas, Josefina María. "Mujeres, animales y soberanía alimentaria: hacia una racionalidad ético-económica en favor de la vida. Universidad Michoacana de San Nicolás de Hidalgo". En: Revista de Filosofía y Filosofía de la Cultura 15(30). Morelia: 2014, 135-182.

Contreras, Jesús y Gracia, Mabel. Alimentación y cultura: perspectivas antropológicas. Barcelona: ed. Aries, 2005a.

- Cuerpo, dieta y cultura. En Alimentación y Cultura: Perspectivas Antropológicas, 256-290. Barcelona: Editorial Arieló, 2005b.

Contreras Hernández,Jesús. "La modernidad alimentaria: entre la sobreabundancia y la inseguridad”. En: Revista Internacional de Sociología 40 (2005): 109-132. 
Davidson College. ¿Conservar o Consumir? El impacto de las Áreas Naturales Protegidas en la Seguridad y la Soberania Alimentaria de la Gente Indigena en Madre de Dios, Perú / conserve or Consume? The impact of Protected Natural Areas on Security and the Food Sovereignty of Indigenous People in Madre de Dios, Peru. Adde Sharp SIT Study Abroad, 2018.

Entrena Duran, Francisco. "Globalización, identidad social y hábitos alimentarios". En: Revista de Ciencias Sociales (Cr) 1(119). San Jose: Universidad de Costa Rica, 2008, 27-38.

Fernández Núñez, Lissette. “¿Cómo analizar datos cualitativos?”: En: Institut de Ciències de l'Educació Secció de Recerca (7) (2006): 1-13.

García Cardona, Mercedes; Pardio Lopez, Jeannette; Arroyo Acebedo, Pedro y Fernández García, Victoria. "Dinámica Familiar y su relación con hábitos alimentarios”. En: Estudios sobre las Culturas Contemporáneas 14(27). México: Universidad de Colima, 2008, 09-46.

González Gil, Teresa y Cano Arana, Alejandro. "Introducción al análisis de datos en investigación cualitativa: Tipos de análisis y proceso de codificación (II)". En: Nure Investigación, (45) (2010): 1-10.

Heinisch, C. Soberanía. Un análisis del concepto. Editores Francisco Hidalgo, Pierril Lacroix y Paula Román. Ecuador: SIPAE, 2013, 11-36.

Lee Gwenyth; Paredes Olortegui Maribel; Rengifo Pinedo Sylvia; Ambikapathi Ramya; Peñataro Yori Pablo; Kosek Margaret y Caulfield, Laura. "Feeding practices in the Peruvian Amazon: implications for programs to improve feeding”. En: Revista Panam, Salud Publica 36(3) (2014): 150-157.

Maganto, Carmen; Garaigordobil, Maite y Kortabarria, Lorea. “Variables antropométricas, hábitos y dietas alimentarias en adolescentes y jóvenes: diferencias en función de sexo”. En: Acción Psicología 13(2) (2016): 89-100.

Morales Camelo, Natay y Paipilla Fernández, Stephany. "La alimentación imaginada: Una cartografía de la seguridad alimentaria de las comunidades indígenas del municipio del Mitu, Vaupés, Colombia”. En: Revista Ciencias Humanas. Brasil: UNITAU, Taubate/SP, 2020, 56-71.

Mintz, Sidney W. La comida en relación con los conceptos de poder. En Sabor a Comida, Sabor a Libertad: Incursiones en la Comida, la Cultura y el Pasado, 39-56. México: Ediciones de la Reina Roja, 2003. 
Oseguera Parra, David. "Comidas peligrosas: la percepción social de la (in) seguridad alimentaria”. En: Estudios sobre las Culturas Contemporáneas, 10(19). México: Universidad de Colima, 2004, 31-51.

Oseguera Parra, David y Esparza Serra, Luis L. "Significado de la seguridad y el riesgo alimentarios entre indígenas purhépechas de México”. En: Desacatos (31) (2009): 115-136.

Oyola Prado, Kirla Fara y Mosqueira Mosqueira, Augusto César. Sistemas mixtos de producción y desarrollo en la comunidad machiguenga de Tivoriari. Tesis para optar grado de licenciatura en Antropología. Universidad Nacional de San Antonio Abad del Cusco. Cusco: 2013.

Peña Venegas, Clara Patricia; Mazorra Valderrama, Augusto; Acosta Muñoz, Luis Eduardo y Perez Rúa, Monica Natalia. Seguridad alimentaria en comunidades indigenas de las amazonas: ayer y hoy. 1ra ed. Bogotá: Instituto Amazónico de Investigaciones Científicas - Sinchi, 2009, 09-139.

Pérez Gil, Sara Elena y Romero J., Gabriella. "Imagen corporal y representaciones alimentarias en mujeres de trece comunidades rurales mexicanas". En: Población y salud en Mesoamérica 15(2) (2018): 01-27.

Ríos Arevalo, Michelly; Camacho Palomino, Edwin. "La agrobiodiversidad en várzea y su función económica en la Amazonía peruana”. En: Scientia Agropecuaria, 7(4). Brasil: Universidad Federal do Oeste do Para, 2016, 377-389.

Rosas Chávez, Nadia Marlene \& Rico Rodriguez, Tyanif. "El papel de las mujeres en la construcción de la soberanía alimentaria". En: Revista de investigación y divulgación sobre los estudios de género 2(21) (2017): 95-118.

Segura García Del Río, Baldomero. "Incidencia de la inseguridad alimentaria en los procesos productivos agroalimentarios" (Editorial). En: Revista Mexicana de Agronegocios 32. México: Sociedad Mexicana de Administración Agropecuaria, 2013, 155-156.

Silva Guerra, Giovanna (comp.). El Rostro Amazónico del Cusco. Cusco: Instituto Nacional de Cultura, 2005.

Silvada da Costa, Marcilene. "Prácticas alimenticias en una comunidad Quilombola de la Amazonía Brasileña”. En: Revista Pueblos y Frontera digital 7(14) (2013): 177-203. 
Terreno, Felicitas. "Una respuesta regional a la (in) seguridad alimentaria”. En: Revista de Investigaciones Agropecuarias 38(2). Buenos Aires: Instituto Nacional de Tecnología Agropecuaria, 2012, 114-118.

Troncoso-Pantola, Claudia; Muñoz-Roa, Mirna; Sotomayor-Castro, Mauricio; Amaya-Plascencia, Juan Pablo; Alarcos-Rivero, Mari y Amaya-Plascencia, Antoni. "Significancia Gastronómica de recetas favoritas en mujeres adultas mayores”. En: Estudios Sociales. Revista de alimentación contemporánea y desarrollo regional, 29(54) (2019): 02-24.

Vargas, Gabriel E.; Espinoza, Giovana; Ruiz, Candy y Rojas, Rosario. "Valor Nutricional de la larva de Rhynchophorus palmarum L.: Comida tradicional en la Amazonía peruana”. En: Revista de la Sociedad Química del Perú, 79(1) (2013): 64-70.

Vargas Tierras, Yadira Beatriz; Prado Beltran, Julia Karina; Nicolalde Cruz, José Rogelio; Casanoves, Fernando; Virginio Filho, Elías De Melo y Viera Arroyo, William Fernando. "Caracterización y rol de los frutos amazónicos en fincas familiares en las provincias de Sucumbíos y Orellana (Ecuador)”. En: Corporación colombiana de investigacion agropecuaria 19(3). Mosqueira: 2018, 485-499.

Viola T., María. "Estudios sobre modelos de consumo: una visión desde teorías y metodologías". En: Centro de Estudios del Sistema Agroalimentario. Buenos Aires: Universidad Nacional de Lomas de Zamora, 2008, 01-09.

Zapata, María Eliza; Rovirosa, Alicia y Carmuega, Esteban. "Urbano y rural: diferencias en la alimentacion de los hogares argentinos según el nivel de ingreso y área de residencia”. En: Revista Salud Colectiva. Argentina: Universidad Nacional de Lanús, 2019, 02-13. 